\title{
A Good Death is Hard to Find
}

\author{
Daniel K. Ting, MD*
}

"Is this the last night?"

Her husband lies beside us in a post-ictal stupor with drool forming at his lower lip. Jack is hooked up to a cardiac monitor, has two intravenous lines and is breathing oxygen through a noninvasive positive pressure mask. A staccato interruption of mechanized alarms breaks the silence. The wife, decades younger, dresses in a tan and mustard fast food uniform, smelling faintly of cooking oil. She tells me Jack has been suffering the past two years-horribly, daily, progressively-and has in more lucid states pleaded against any more medical interventions. Coming to the Emergency Department was a panicked, desperate decision.

"Yes," I say, "This could be the last night."

The wife leans over the hospital bed to kiss Jack on his cheek but most of the space is blocked by the oxygen mask so she settles for his forehead instead.

Half an hour earlier, I was working in the ICU when my pager went off: one of the Emergency Physicians told me about a patient he wanted admitted to the unit. A sixty-year-old man named Jack had arrived in respiratory failure. A lifetime cigarette smoker, his endstage emphysema had led to several hospital admissions over the past year. My review of his past medical history is grim. I note three prior myocardial infarctions with an ejection fraction barely making double digits. His cognition has deteriorated from recurrent strokes, his kidney function is poor, and his blood sugar readings have been chaotic. The Emergency Physician relates that Jack just had a hypoxic seizure in the trauma bay, which only aborted with medication.

I call my ICU attending on the phone to review the case. On the third ring she picks up. Her voice comes in a little rough and I guess I am waking her from sleep. Midway through my patient presentation, she says:
"This is a man who should never have been brought to hospital and should have been allowed to die at home. We are not going to be aggressive with him. If he arrests during the night I want you to step back and push the morphine."

"What if he seizes again?" I ask.

"Push the morphine," she says, and the silence that follows means I am on my own.

Jack is transferred upstairs to the ICU. Speaking with his new nurse, I explain our objective of palliation. Jack looks as unresponsive as before, and I can hear him wheeze through the oxygen mask. Nevertheless, I convince myself that there has not been a significant clinical change and half-stumble to the on-call room, exhausted. My digital wristwatch reads 0235 .

Before long, my pager blares me from a pre-sleep state not to be confused with rest. Jack is seizing again. I slip on my running shoes, find my stethoscope and head back towards the ICU, my pupils constricting tenderly against the overhead fluorescents. Treating seizures is routine and takes minimal mental effort, but my mind struggles with less algorithmic issues. If I save this man, what am I saving? If I let him go, is it really my decision to make? Less than a year removed from wearing the short white coat of a clerk, the mantle of "Doctor" feels especially heavy tonight.

I reach Jack's bedside and I do the hardest thing, which is to do nothing. Seizures often self-terminate within minutes and I hold out for that. The nurse and I stand there, watching. Jack's right arm twitches and his eyes roll back. Five, then ten minutes pass. The convulsions grow more violent, increase their amplitude and soon encompass the whole arm. The bedsprings rattle. Torn apart by indecision I ask myself whether one has the right to die in a good way. The answer is that I do not know a good way but I do know a few

From the *Department of Emergency Medicine, University of British Columbia, Kelowna BC.

Correspondence to: Dr. Daniel Ting, Department of Emergency Medicine, Kelowna General Hospital, 2312 Pandosy Street, Kelowna BC V1Y 1T3; Telephone: (250) 980-1310; Email: daniel.ting@alumni.ubc.ca

(C) Canadian Association of Emergency Physicians

CJEM 2017;19(5):390-391

DOI 10.1017/cem.2016.356 
wrong ways and this is one of them. I meet the nurse's eye that has been trying to catch mine and I tell her, tentatively at first but then more firmly, to start the full treatment, intravenous benzodiazepines and antiepileptic medications.

The trembling of Jack's body decreases until it is only his abdomen vibrating back and forth. I stare at it for what seems like a long time before I realize he has been breathing like that the whole time; he has just been so dyspneic that his abdominal muscles are fully engaged to take in oxygen. He is anguishing, in a different way, still. Whether Jack looks more uncomfortable now or when he was seizing, I do not know. He remains the same man with multi-organ failure and incurable lungs.
The next morning, I am expecting my attending to berate me at bedside rounds for prolonging unnecessary suffering. To my surprise, she taps me on the shoulder, gives me a small, knowing nod, and says, "You did the right thing."

I question if she means it or if that is just the thing you say, but when I go home after the 30-hour shift it only takes me a few moments to fall asleep. And now, when I reflect back, I wonder if that is the reason we choose to do something when we are beyond saving lives.

\section{Competing interests: None.}

Keywords: emergency medicine, humanities, palliative care, critical care 